\title{
Posicionamento da ASBAI sobre dose adicional e intercambialidade de vacinas contra a COVID-19
}

\author{
ASBAI position statement on additional dose and interchangeability of COVID-19 vaccines
}

\author{
Pedro Giavina-Bianchi ${ }^{1}$, Lorena de Castro Diniz² ${ }^{2}$ Ekaterini Goudouris ${ }^{3}$, \\ Ana Karolina Barreto Berselli Marinho', Dewton de Moraes Vasconcelos ${ }^{4}$, \\ Norma de Paula Motta Rubini ${ }^{5}$, Emanuel Sarinho 6
}

\begin{abstract}
A covID-19 (Coronavirus Disease 2019) causada pelo SARS-CoV-2 (Severe Acute Respiratory Syndrome Coronavírus-2) foi declarada uma pandemia pela Organização Mundial da Saúde (OMS) em 11 de março de 2020 e tem sido uma catástrofe que assola o mundo com efeitos devastadores na saúde pública, economia e sociedade. Vivenciamos não apenas a pandemia de COVID-19, mas também uma infodemia. Existem muitas publicações importantes, mas outras discordantes, polêmicas e inconclusivas, além da disseminação de informações falsas, irresponsáveis e oportunistas pelo mundo. Nesse contexto a Associação Brasileira de Alergia e Imunologia publica seu posicionamento em relação à administração de dose adicional no esquema vacinal originariamente estudado contra a COVID-19 e quanto à intercambialidade entre as diferentes vacinas.

As vacinas contra o SARS-CoV-2 têm se mostrado eficazes na prevenção da COVID-19 em ensaios clínicos randomizados e em estudos de vida real, principalmente no que se refere a infecções graves associadas à internação hospitalar e mortes, adquirindo papel principal e sendo imprescindível para
\end{abstract}

o controle da pandemia. Há evidências científicas robustas para todos os imunizantes registrados $\mathrm{e}$ utilizados no Brasil ${ }^{1-4}$.

São propriedades do sistema imune a especificidade, a geração de memória e a autorregulação. A resposta imune se regula e naturalmente vai reduzindo após a cessação do estímulo que a desencadeou, podendo deixar de ser protetora com o tempo. Paralelamente a essas características, alguns grupos, como os idosos e os pacientes com imunodeficiências primárias ou secundárias, têm menor capacidade de desenvolver respostas imunes protetoras e podem ter respostas vacinais inadequadas, ficando suscetíveis a infecções. Estudo brasileiro em população idosa evidenciou que a proteção da vacina CoronaVac foi baixa até a conclusão do regime de duas doses e que a eficácia da vacina diminuiu com o aumento da idade ${ }^{5}$. Há ainda outros grupos, como os profissionais de saúde, que apresentam maior risco de infecções devido à maior exposição ao SARS-CoV-2 ${ }^{6}$.

Estudos recentes demonstraram a diminuição da eficiência da vacinação anti-COVID-19 em decorrência da redução da resposta imune, surgimento de

1. Comissão COVID-19 ASBAI. Disciplina de Imunologia Clínica e Alergia, FMUSP - São Paulo, SP, Brasil.

2. Comissão COVID-19 ASBAI. Centro de Referência de Imunobiológicos Especiais - Goiânia, GO, Brasil.

3. Comissão COVID-19 ASBAI. Departamento de Pediatria, FM da UFRJ - Rio de Janeiro, RJ, Brasil.

4. Comissão COVID-19 ASBAI. Departamento de Dermatologia do HCFMUSP - São Paulo, SP, Brasil.

5. Diretora Científica ASBAI. Disciplina de Alergia e Imunologia, Escola de Medicina e Cirurgia, UNIRIO - Rio de Janeiro, RJ, Brasil.

6. Presidente ASBAI. Centro de Ciências da Saúde, Departamento Materno-Infantil, Universidade Federal de Pernambuco - Recife, PE, Brasil. 
cepas variantes e menor distanciamento social e uso de medidas protetoras ${ }^{7,8}$. Observou-se uma redução da eficácia das vacinas de mRNA (Pfizer e Moderna) em relação à prevenção de infecções sintomáticas pelo coronavírus de cerca de $95 \%$ para $65 \%$ em trabalhadores da área da saúde ${ }^{8}$.

Outra discussão referente à intercambialidade de vacinas se iniciou com a escassez e a dificuldade de gestão devido à indisponibilidade dos diferentes imunizantes, assim como da ocorrência de efeitos adversos à determinada vacina. $O$ conceito da intercambialidade das vacinas contra o SARS-CoV-2 consiste na possibilidade de troca dos diferentes tipos de vacinas, inclusive de diferentes plataformas tecnológicas, sem afetar a eficácia e segurança do esquema vacinal. Estudo de grande relevância analisando o tema mostrou que doses heterólogas (diferentes) combinadas das vacinas ChAdOx1 (AstraZenecaOxford) e BNT162b2 (Pfizer) foram seguras e superiores em alguns parâmetros da resposta imune humoral e celular na comparação com duas doses homólogas (iguais) da mesma vacina, ChAdOx1 ou BNT162b29. Associadas a esse estudo, outras pesquisas mostraram que as únicas vacinas que desencadearam uma razoável resposta imune após a primeira dose foram as de mRNA (Pfizer, Moderna), em virtude do efetivo treinamento da resposta inata ${ }^{1-5}$. Portanto, no momento, a intercambialidade de vacinas para apenas uma dose adicional ao esquema vacinal inicial tem maior embasamento para as vacinas de mRNA.

Especula-se que doses adicionais no esquema vacinal contra a COVID-19 e que a combinação de imunizantes possam aumentar a eficiência da vacinação na prevenção da COVID-19. Hoje, à luz das evidências científicas, o posicionamento da Associação Brasileira de Alergia e Imunologia preconiza a realização de uma dose adicional das vacinas anti-SARS-CoV-2 nos grupos que apresentam maior chance de adquirirem a COVID-19, ou por não estarem imunes após o esquema vacinal inicial, especificamente os idosos e pessoas com imunodeficiências primárias ou secundárias, ou por serem profissionais da saúde em contato com pacientes potencialmente infectados com cargas virais elevadas e com variantes mais virulentas. Em relação à intercambialidade das vacinas, sugerimos que a dose adicional seja feita preferencialmente com uma vacina de mRNA da proteína S (Pfizer, Moderna). Conduta alternativa seria a administração de uma dose adicional de vacina homóloga (igual) àquelas utilizadas na imunização inicial. Estudos começam a ser divulgados mostrando aumento de proteção com a terceira dose da vacina BNT162b2 (Pfizer) ${ }^{10,11}$ e as pesquisas com as demais vacinas estão em andamento. Apesar de mais estudos serem necessários para determinar a proteção conferida pela terceira dose e, principalmente, seu efeito na doença grave, a indicação dessa dose adicional não poderá esperar.

\section{Referências}

1. Polack FP, Thomas SJ, Kitchin N, Absalon J, Gurtman A, LockhartS, C4591001 Clinical Trial Group. Safety and Efficacy of the BNT162b2 mRNA Covid-19 Vaccine. N Engl J Med. 2020;383(27):2603-15. doi: 10.1056/NEJMoa2034577.

2. Ramasamy MN, Minassian AM, Ewer KJ, Flaxman AL, Folegatti PM, Owens DR; Oxford COVID Vaccine Trial Group. Safety and immunogenicity of ChAdOx $1 \mathrm{nCoV}-19$ vaccine administered in a prime-boost regimen in young and old adults (COV002): a single-blind, randomised, controlled, phase 2/3 trial. Lancet. 2021;396(10267):1979-93. doi: 10.1016/S01406736(20)32466-1.

3. Jara A, Undurraga EA, González C, Paredes F, Fontecilla T, Jara $G$, et al. Effectiveness of an Inactivated SARS-CoV-2 Vaccine in Chile. N Engl J Med. 2021;385(10):875-84. doi: 10.1056/ NEJMoa2107715.

4. Chodick G, Tene L, Rotem RS, Patalon T, Gazit S, Ben-Tov A, et al. The effectiveness of the TWO-DOSE BNT162b2 vaccine: analysis of real-world data. Clin Infect Dis. 2021:ciab438. doi: 10.1093/cid/ ciab438.

5. Ranzani OT, Hitchings MDT, Dorion M, D'Agostini TL, de Paula RC, de Paula OFP, et al. Effectiveness of the CoronaVac vaccine in older adults during a gamma variant associated epidemic of covid- 19 in Brazil: test negative case-control study. BMJ. 2021;374:n2015. doi: 10.1136/bmj.n2015.

6. Costa SF, Giavina-Bianchi P, Buss L, Mesquita Peres $\mathrm{CH}$, Rafael MM, Dos Santos LGN, et al. SARS-CoV-2 seroprevalence and risk factors among oligo/asymptomatic healthcare workers(HCW): estimating the impact of community transmission. Clin Infect Dis. 2020 Dec 13:ciaa1845. doi: 10.1093/cid/ciaa1845.

7. Madhi SA, Koen AL, Izu A, Fairlie L, Cutland CL, Baillie V; Wits VIDA COVID team. Safety and immunogenicity of the ChAdOx $1 \mathrm{nCoV}-19$ (AZD1222) vaccine against SARS-CoV-2 in people living with and without HIV in South Africa: an interim analysis of a randomised, double-blind, placebo-controlled, phase $1 \mathrm{~B} / 2 \mathrm{~A}$ trial. Lancet HIV. 2021 Sep;8(9):e568-e580. doi: 10.1016/S2352-3018(21)00157-0.

8. Keehner J, Horton LE, Binkin NJ, Laurent LC, Pride D, Longhurst CA, et al. Resurgence of SARS-CoV-2 Infection in a Highly Vaccinated Health System Workforce. N Engl J Med. 2021. doi: 10.1056/ NEJMc2112981.

9. Hillus D, Schwarz T, Tober-Lau P, Vanshylla K, Hastor H, EICOV/ COVIM Study Group. Safety, reactogenicity, and immunogenicity of homologous and heterologous prime-boost immunisation with ChAdOx1 nCoV-19 and BNT162b2: a prospective cohort study. Lancet Respir Med. 2021:S2213-2600(21)00357-X. doi: 10.1016/ S2213-2600(21)00357-X.

10. Patalon T, Gazit S, Pitzer VE, Prunas O, Warren JL, Weinberger DM. Short term reduction in the odds of testing positive for SARSCoV-2; a comparison between two doses and three doses of the BNT162b2 Vaccine. medRxiv doi: https://doi.org/10.1101/2021.0 8.29.21262792.

11. Bar-On YM, Goldberg Y, Mandel M, Bodenheimer O, Freedman L,Kalkstein N, et al. BNT162b2 vaccine booster dose protection: A nationwide study from Israel. https://www.gov.il/BlobFolder/reports/ vaccine-efficacy-safety-follow-up-committee/he/files_publications_ corona_booster-27082021.pdf. 